\title{
Kinetics of singlet and triplet excitons in a wide-band-gap copolymer
}

\author{
M. A. Loi \\ Dipartimento di Fisica and INFM, Università di Cagliari, I-09042 Monserrato (CA), Italy \\ C. Gadermaier, E. J. W. List, and G. Leising \\ Institut für Festkörperphysik, Technische Universität Graz, A-8010 Graz, Austria \\ W. Graupner \\ Department of Physics, Virginia Tech, VA-24060 Blacksburg, Virginia \\ G. Bongiovanni and A. Mura \\ Dipartimento di Fisica and INFM, Università di Cagliari, I-09042 Monserrato (CA), Italy \\ J.-J. Pireaux \\ Laboratoire LISE, Facultés Universitaires Notre-Dame de la Paix, B-5000 Namur, Belgium \\ K. Kaeriyama \\ Department of Materials Science, Kyoto Institute of Technology, Kyoto 606, Japan
}

(Received 13 August 1999)

\begin{abstract}
Transient and photomodulation spectroscopy is used in order to determine decay times and densities of both emitting and absorbing species in the wide band-gap semiconductor poly-2,5-diheptyl-1,4-phenylene-alt-2, 5-thienylene (PDHPT). The wide band gap of this material is a consequence of the large twisting of the neighboring constituents of the polymer chain. Transient spectroscopy revealed a monoexponential decay of singlet excitons in PDHPT solutions with a radiative and nonradiative decay time of 1.9 and $1.6 \mathrm{~ns}$, respectively. For the solid film photoluminescence decays biexponentially. This distinct decay behavior in solid state is attributed to the migration of the excitons towards quenching sites favored by the three dimensionality in the bulk material, which hence reduces the photoluminescence quantum yield. Infrared studies suggest that upon photo-oxidation one introduces carbonyl groups in PDHPT, which shortens the decay times by introducing photoluminescence quenching centers. Photomodulation spectroscopy is exploited to determine steady-state density of triplet excitons along with their decay dynamics and we found that the steady-state density of the triplets can be as high as $10^{16} \mathrm{~cm}^{-3}$ in this material. Furthermore, we determined the generation probability of triplets to be $2 \times 10^{-3}$.
\end{abstract}

\section{INTRODUCTION}

Poly - 2, 5 - diheptyl - 1, 4-phenylene - alt - 2, 5-thienylene ${ }^{1}$ (PDHPT) is a regular alternation of high gap units (benzoic) and low gap units (thienylenic) as depicted in Fig. 1. The alkyl side groups are introduced to make the copolymer soluble, increasing consequently the processibility. ${ }^{2,3}$ The side chains do not take part in the $\pi$ bonds, but their steric hindrance induce a considerable inter-ring twisting, giving rise to a substantial reduction of the polymer conjugation length PDHPT has emerged as an attractive material for turquoise light emitting diodes. ${ }^{1}$ Moreover, the large Stokes shift between absorption and emission suggests also its possible use as active medium for laser diodes. However, for effective practical applications of PDHPT as active laser media or other optoelectronic applications, a fundamental knowledge about the luminescence quantum yield, the photoinduced absorption bands and their overlap with the emission spectrum as well as the decay channels and the dynamics of excited states is necessary.

In order to get a more complete knowledge of the optical properties of PDHPT, we have studied dilute solutions and spin-coated films of PDHPT with different spectroscopic techniques. We present experimental data on cw absorption, steady-state photoluminescence (PL), PL quantum yield (QY), time-resolved PL, Fourier transform infrared (FTIR), photoinduced absorption (PIA) as well as doping-induced absorption (DIA).

Our data provide a deeper insight into the influence of the side chains and the alternation of high- and low-gap units on

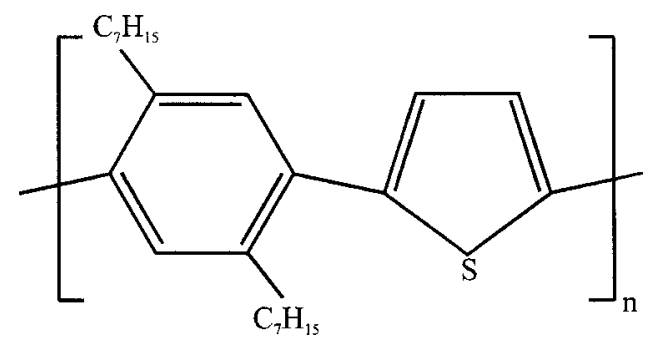

FIG. 1. Chemical structure of poly-2,5-diheptyl-1,4-phenylenealt-2,5-thienylene (PDHPT). 
the electronic levels. We have investigated the influence of interchain interactions and oxidation on the photoexcitation energies and relaxation pathways in this class of copolymers. Furthermore, by means of a profound analysis of the PIA measurements we determine the parameters that control the creation and the dynamics of the triplet states in this material.

\section{EXPERIMENT}

PDHPT was prepared by a Suzuki reaction-the synthesis is reported in Ref. 4.

Thin-film samples for optical measurements were prepared by spin coating from chloroform or toluene solution onto quartz substrates. The absorption spectra were recorded using a Perkin Elmer $\lambda 9$ spectral analyzer. The DIA experiment was performed using gaseous iodine as dopant. The PL spectra have been recorded with a Shimadzu RF-5301PC spectrofluorophotometer. The quantum yield of the film was measured with an integrating sphere as described in Ref. 5, while for the solution it was obtained using coumarin 120 as a reference solution as described in Ref. 6 .

For the PIA measurements the sample was excited by an 351.1/363.8- $\mathrm{nm} \mathrm{Ar}^{+}$laser beam, which was chopped mechanically with a frequency of about $89 \mathrm{~Hz}$ providing the reference for the lock-in amplifier (5210, EG\&G Princeton). For the interpretation we plot the spectral dependence of the ratio of the photogenerated change in sample transmission $\Delta T$ normalized to the transmission $T$ of the film. Since the sample is strongly luminescent the measured $\Delta T$ signal also contains a PL component, which can easily be determined by taking $\Delta T$ with the probe beam "off" - this signal is then subtracted from the $\Delta T$ measurement with the probe beam "on." The sample was mounted within an optically accessible cryostat under dynamic vacuum kept at a constant temperature of $90 \mathrm{~K}$. A $250 \mathrm{~W}$ tungsten halogen lamp provided the light for the transmission measurement. For the recording of the PIA spectrum a grating monochromator and a silicon photodiode were used.

Spontaneous emission for time resolved measurements was excited by the fourth harmonic of a pulse compressed Nd: YAG $(4.66 \mathrm{eV})$ delivering 4 ps pulses, or second harmonic $(3.20 \mathrm{eV})$ of a synchronously pumped dye laser delivering $0.5 \mathrm{ps}$ pulses. The signal was recorded by a Hamamatsu optical sampling oscilloscope with 20 ps of time resolution.

FTIR measurements were performed with spin coated films on silicon substrates using a Bomem Michelson spectrometer MB 102.

\section{RESULTS AND DISCUSSION}

\section{A. Photoluminescence and absorption}

Figure 2 shows the absorption and cw-PL emission spectra of the spin-coated PDHPT film and a dilute PDHPT solution. The shape of these spectra is rather similar, both spectra do not show any vibrational structure. The absorption maxima of the thin film and the solution are at 3.90 and at $3.75 \mathrm{eV}$, respectively. The PL spectra peak at 2.70 and at $2.73 \mathrm{eV}$. The considerable Stokes shift can be ascribed to the conformational changes with a more rigid planar structure

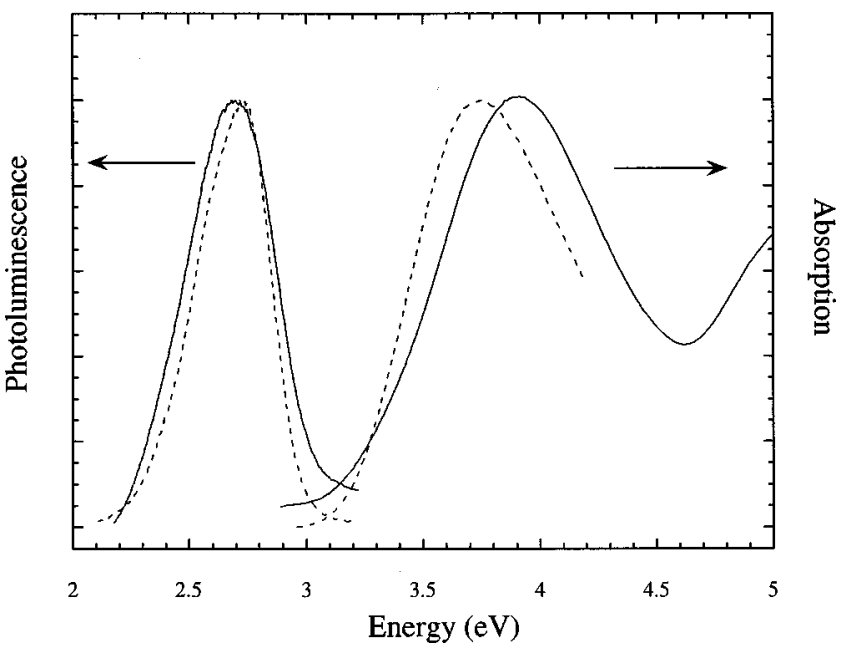

FIG. 2. Absorption and photoluminescence emission spectra of thin film (solid line) and solution (dashed line).

occurring after photoexcitation, ${ }^{7,8}$ and to a possible exciton migration from short- to long-conjugation segments in the copolymer prior to recombination.

PDHPT is a block copolymer made up by the alternate sequence of thiophene and phenyl rings. Theoretical works on copolymers composed by alternating low and high bandgap materials suggest that the band gap of the copolymer is the weighted average of the gap values of the parent polymers. ${ }^{9}$ However, the absorption edge of PDHPT, is found at $3.18 \mathrm{eV}$ both for film and solution, it is therefore located at higher energies than that of unsubstituted polyparaphenylene $(\sim 2.7 \mathrm{eV},[10])$ and unsubstituted polythiophene $(\sim 1.9 \mathrm{eV},[11])$. The high-optical gap also rules out that the electronic levels of PDHPT behave like found for typical quantum well structures. ${ }^{12,13}$ Therefore, the high-absorption edge of PDHPT cannot be explained by "band-gap averaging" of two unsubstituted parent polymers. Looking at monosubstituted PPP and disubstituted PPP one finds absorption edges at 3.1 (Ref. 2) and $3.4 \mathrm{eV}$ (Ref. 8), respectively. Following the approach of Meyers, Heeger, and Bredas $^{9}$ of calculating the band gap via arithmetic averaging we get $(3.4 \mathrm{eV}+1.9 \mathrm{eV}) / 2=2.7 \mathrm{eV}$ for the copolymer consisting of disubstituted phenyl units and unsubstituted thiophene units. This suggests that there is an additional mechanism increasing the band gap. Theoretical calculations yield inter-ring torsion angles between the thiophene and the phenylene units as high as $50^{\circ}{ }^{1}$ We suggest that the unexpected high-energy gap is due to the reduction of the $\pi$ conjugation induced by the rather high twisting of the molecular backbone, which is mainly caused by the steric hindrance due to the alkyl groups. ${ }^{14}$ Hence, the present absorption data suggest that the effect induced by the large inter-ring torsions on the electronic properties of PDHPT is much more pronounced than the expected band-gap lowering due to presence of the thiophene units.

\section{B. Time-resolved photoluminescence}

Figure 3 shows the PL decay of a dilute solution and a thin film. The solution exhibits an almost monoexponential decay with a time constant $\tau_{P L}$ of 860 ps. The combined 


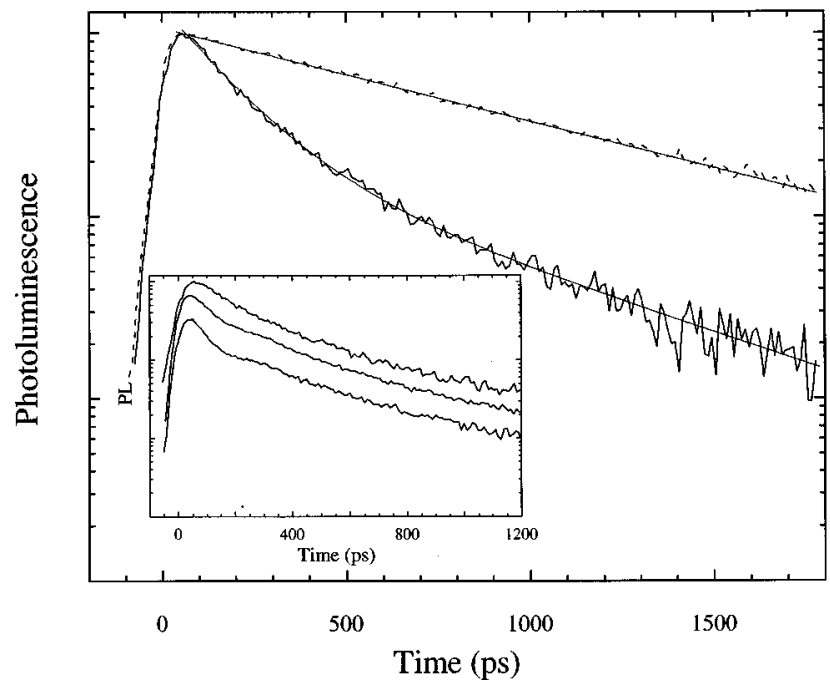

FIG. 3. Decay of the photoluminescence in solution (dashed line) and thin film (solid line). The two smooth solid lines are a monoexponential fits to the solution decay and a biexponential fit to the thin-film decay, respectively. Insert: Decay of photoluminescence for different UV irradiation time. The exposure degree increases from the top to the bottom. The decays are displaced vertically for clarity of presentation.

measurements of PL decay and emission QY can be used to estimate the radiative lifetime $\tau_{r}$ of PDHPT. The QY was determined to be $45 \%$ for dilute solutions and $20 \%$ in the thin film. If one assumes that the photoexcitation process creates singlet excitons, the quantum efficiency for the solution $\mathrm{QY}_{s}$ can be expressed as $b \tau_{P L} / \tau_{r},{ }^{15}$ where $b$ represents the fraction of absorbed photons leading to the generation of singlet excitons. By reasonably assuming $b=1,{ }^{15}$ we find that the radiative lifetime in solution is $1.9 \mathrm{~ns}$.

The photoluminescence decay of the thin film is not exponential, indicating a more complex relaxation dynamics. The transient emission can be fitted by a biexponential law with an initial fast decay, $\tau_{P L 1}=160 \mathrm{ps}$ and a slower one, $\tau_{P L 2}=630 \mathrm{ps}$. Such a behavior suggests that different decay pathway contribute to the emission, further we believe that the same excited species is involved in the decay of the solution and of the thin film. We attribute the two decay times in the solid film to a different decay pathway for the same photoexcited species as follows. Since $\tau_{P L 2}$ is very similar to the solution decay time, the slower emission is attributed to excitons' motion confined within a single chain. $\tau_{P L 1}$ has to be related to the interchain migration within the threedimensional network in the solid state. The higher mobility of excitons favors the migration towards quenching sites enhancing the nonradiative decay rate, which in turn can account for the faster decay contribution to the PL observed in the solid state. ${ }^{16,17}$

It is possible to estimate the quantum efficiency of the film $\mathrm{QY}_{f}$ by using the PL decay data, with the aim to compare it with the $\mathrm{QY}_{f}$ directly measured by means of the integrating sphere. ${ }^{18}$ In fact the $\mathrm{QY}=N_{e m} / N_{a b s}$; where $N_{e m}$ and $N_{a b s}$ are the number of emitted and absorbed photons, respectively. $N_{e m}$ can be calculated from the time-resolved measurements by integrating the decay curve, $N_{e m}$ $=$ const $\int \operatorname{PL}(t) d t$. If we assume that the same species is ex-

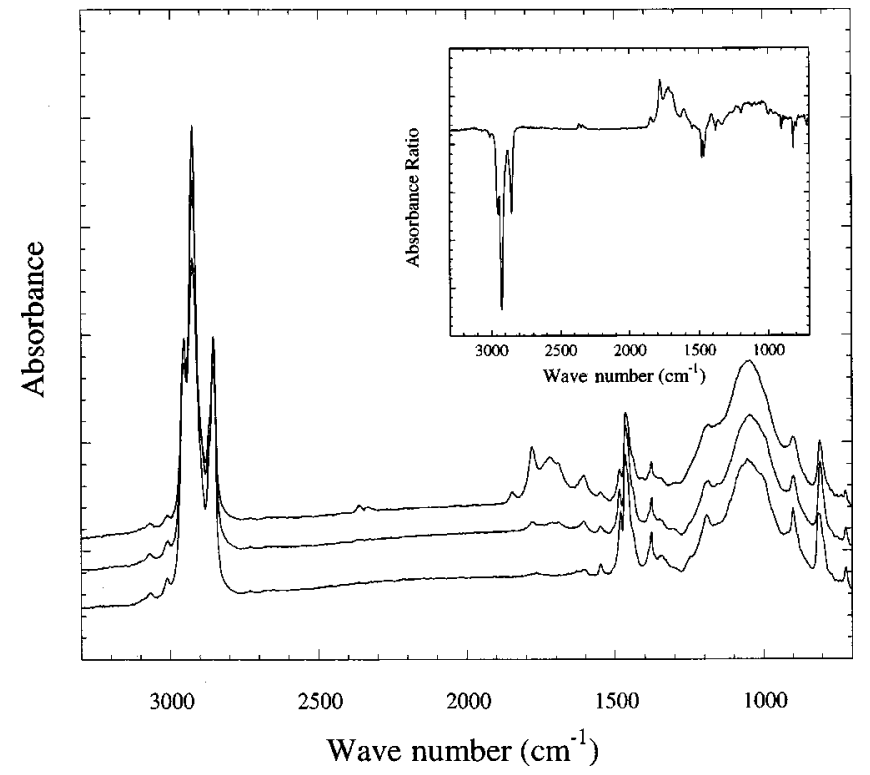

FIG. 4. Infrared spectra of thin-film sample. From the bottom to the top, sample not exposed to UV, one minute and three minutes of UV exposure. The spectra are displaced vertically for clarity of presentation. Insert: Ratio between the IR spectra of the most oxidized sample and the not oxidized one.

cited in solution and in film, we can reasonably assume an equal radiative decay rate and thus the same proportional factor in the previous equation. In this way, it is possible to obtain a quantitative estimation of the $\mathrm{QY}_{f}$ by integrating the decay of the photoluminescence for the solution and the thin film $\mathrm{QY}_{f}=\mathrm{QY}_{s} \quad\left(N_{f, e m} / N_{s, e m}\right)=0.45 \times 0.4 \sim 18 \%$; with $N_{f, e m}$ and $N_{s, e m}$ being the time-integrated number of emitted photons for the film and solution respectively.

The value obtained is in good agreement with the experimental result $(\sim 20 \%)$, supporting our interpretation.

Finally, studying the decay of the photoluminescence for photo-oxidized thin films also strongly supports the proposed scenario. In fact, the faster decay time component is drastically shortened upon increasing the density of quenching centers, by illuminating the sample on air with UV light ${ }^{19}$ as shown in the insert of Fig. 3. In fact we observe an evident decrease of the initial decay time from 160 to $60 \mathrm{ps}$ with increasing UV irradiation time, while the slow decay, attributed to excitons that cannot reach quenching centers within their lifetime $\tau_{P L 2}$, is almost unchanged (roughly $600 \mathrm{ps}$ ). Moreover, it is important to point out that the faster dynamics for the oxidized sample is accompanied by a drastic reduction of the quantum efficiency.

\section{FTIR spectra}

The FTIR spectra have been used to study the UV exposure induced chemical modifications occurring in PDHPT in order to identify the nature of defect acting as PL quenching sites. ${ }^{19,20}$ Figure 4 shows the FTIR spectra for the sample nonirradiated and for two different irradiation times. We note that x-ray photoemission spectroscopy (xps) measurements indicate that pristine films are free of impurities. ${ }^{21}$ As a consequence of the UV irradiation, new absorption modes appear in the region of $1700 \mathrm{~cm}^{-1}$. We interpret these new 


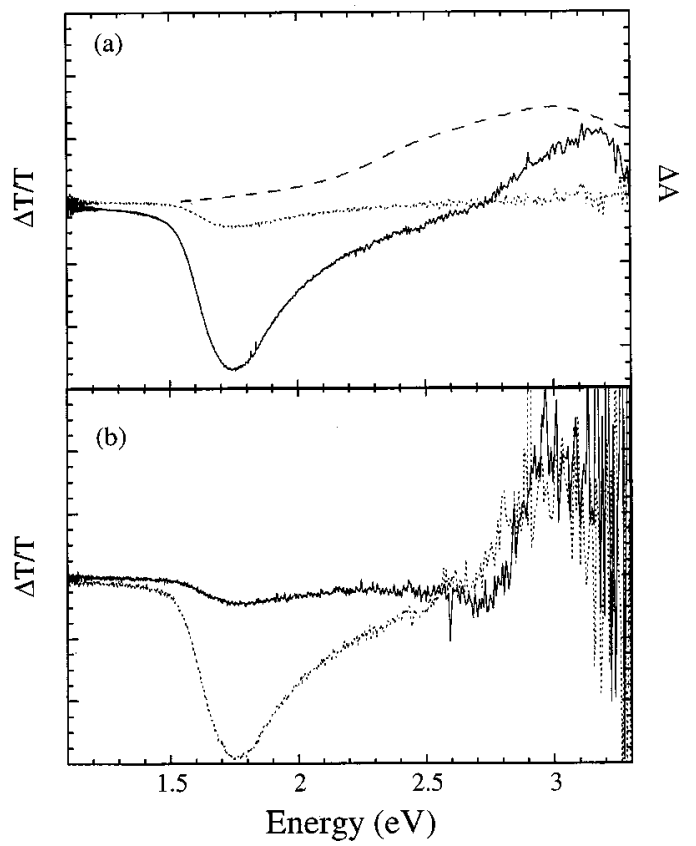

FIG. 5. Photoinduced absorption spectrum at different chopper frequency (a) $9-\mathrm{Hz}$ spectrum in phase with the excitation (solid line) and $90^{\circ}$ out of phase (dotted line). Difference between undoped and doped absorption spectra (dashed line). (b) 900-Hz spectrum in phase with the excitation (solid line) and $90^{\circ}$ out of phase (dotted line).

lines as related to the formation of carbonyl groups $(\mathrm{C}=\mathrm{O}){ }^{19,20,22}$ The insert of Fig. 4 shows that while the band due to the carbonyl group increases, other FTIR modes decrease. The features at $\sim 2950$ and $\sim 1450 \mathrm{~cm}^{-1}$ appear be the most affected by oxidation. We assign the band at $\sim 2950$ $\mathrm{cm}^{-1}$ to the $\mathrm{C}-\mathrm{H}_{2}$ and $\mathrm{C}-\mathrm{H}_{3}$ stretching mode of the alkyl chains ${ }^{23}$ while the region of $\sim 1450 \mathrm{~cm}^{-1}$ is known to be active both for $\mathrm{C}-\mathrm{C}$ and $\mathrm{C}=\mathrm{C}$ stretching for thiophene rings. ${ }^{20,24}$ The decrease of the former two bands suggest that photo- and thermal-oxidation breaks of the $\mathrm{C}-\mathrm{H}$ bonds of the alkyl side groups as well as the $\mathrm{C}-\mathrm{C}$ and $\mathrm{C}=\mathrm{C}$ bonds in the thiophene rings.

The emerging of carbonyl groups, which are known for to break the conjugation length and act as PL quenching sites $^{19,20}$ is in agreement with the findings from timeresolved measurements.

\section{Photomodulation spectroscopy}

In order to study the nature of the nonemissive photoexcited entities in PDHPT, cw PIA at different modulation frequencies $(9$ and $900 \mathrm{~Hz}$ ) and DIA measurements have been performed. Figure 5(a) shows the PIA spectra at a modulation frequency of $9 \mathrm{~Hz}$, and the DIA spectrum, defined as the difference between the absorption after doping and the absorption of the pristine sample. ${ }^{25}$ The negative PIA signal is an indication for the generation of a new absorption band caused by the pump generation of new excited states, the positive PIA signal or photobleaching (PB) is a decrease of the absorption because of the partial depopulation of the ground state after the pump excitation. In Fig. 5(b), we show the PIA spectra at a modulation frequency of about $900 \mathrm{~Hz}$. The PIA spectra both at 9 and $900 \mathrm{~Hz}$ display a pronounced peak at $1.75 \mathrm{eV}$, the PB peak is at 3.18 and $3.00 \mathrm{eV}$ for 9 and $900 \mathrm{~Hz}$, respectively, both are located in the tail of the absorption spectrum, which has its peak a $3.9 \mathrm{eV}$. The position of the PB at $900 \mathrm{~Hz}$ is obscured due to the noise in this particular spectral region introduced by the low probe beam intensity, grating efficiency, silicon detector sensitivity and the PL correction. Supported by the fact that the doping experiments with iodine do not exhibit any contribution of charged states in the spectral region of $1.75 \mathrm{eV}$, we attribute the main peak at $1.75 \mathrm{eV}$ to a dipole allowed $T_{1} \rightarrow T_{n}$ absorption. The triplet excitons in PDHPT are mainly formed by intersystem crossing from the photoexcited singlet state, a process whose efficiency is enhanced by the presence of the sulphur in the thiophene unit. ${ }^{7,26}$ Furthermore, we have to point out that the energy of the $T_{1} \rightarrow T_{n}$ transition in PDHPT is higher than in polythiophene and polyparaphenylene, so that the observed blue shifted triplet-triplet transition provides further evidence for the reduced $\pi$ conjugation induced by the steric hindrance of the alkyl groups. In fact, the transition energy of the triplet-triplet absorption increases with the decrease of conjugation both for thiophenes and for phenyls as summarized in Table I.

The photoinduced absorption peak at $900 \mathrm{~Hz}$ is one order of magnitude less intense than that at $9 \mathrm{~Hz}$. The $T_{1} \rightarrow T_{n}$ absorption peak at low frequency is in phase with the excitation but is out of phase at high frequency. The PB behaves differently; it is in phase with the excitation at $9 \mathrm{~Hz}$ but it exhibits both an in and out of phase component at $900 \mathrm{~Hz}$, which is an indication of the presence of two different components in the PB.

By means of the PIA experiment, it is possible to determine the steady state triplet density $N_{T}$ in PDHPT. In general, absorption is described by Lambert Beer's law

TABLE I. Energy positions for the peak of the triplet-triplet transition and the ground-state absorption edge of unsubstituted thiophene and phenylene based molecules with different chainlengths.

\begin{tabular}{|c|c|c|c|c|}
\hline \multirow[t]{2}{*}{ Number of rings } & \multicolumn{2}{|c|}{ Triplet energy $(\mathrm{eV})$} & \multicolumn{2}{|c|}{ Absorption edge $(\mathrm{eV})$} \\
\hline & phenyls & thiophenes & phenyls & Thiophenes \\
\hline 3 & 2.48 (Ref. 34) & $2.70^{*}$ (Ref. 35) & 3.76 (Ref. 33) & $3.00^{\mathrm{a}}$ (Ref. 35) \\
\hline 4 & 2.35 (Ref. 34) & $2.21 *$ (Ref. 35) & 3.44 (Ref. 33) & $2.75^{\mathrm{a}}$ (Ref. 35)-2.00 (Ref. 36) \\
\hline 5 & & $1.95^{*}($ Ref. 35$)$ & & $2.58^{\mathrm{a}}$ (Ref. 35)-2.10 (Ref. 36) \\
\hline 6 & 1.78 (Ref. 37) & $1.80^{*}$ (Ref. 7)-1.40 (Ref. 7) & 3.00 (Ref. 15) & $2.43^{\mathrm{a}}(\operatorname{Ref} .35)-2.25(\operatorname{Ref} .36)$ \\
\hline$\infty$ & 1.70 (Ref. 25) & $1.55^{*}($ Ref. 7)-1.25 (Ref. 7) & 2.7 (Ref. 10) & $1.9($ Ref. 11$)$ \\
\hline
\end{tabular}

${ }^{\mathrm{a}}$ Sample in solution. 


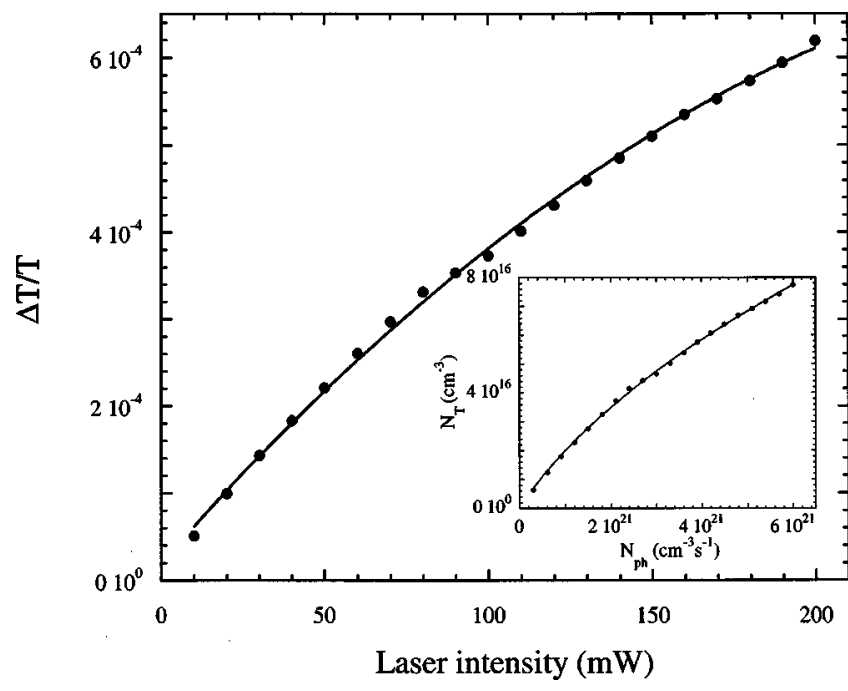

FIG. 6. Photoinduced absorption signal at $1.75 \mathrm{eV}$ (in phase at 9 $\mathrm{Hz}$ ) as function of the pump intensity. The continuous line is a guide to the eye. Insert: Calculated triplet density versus absorbed photons, the continuous line is the fitting with Eq. (7).

$$
I=I_{0} \exp (-\alpha d)
$$

where $I_{0}$ and $I$ are the light intensity incident on the sample and observed at a thickness $d$ of the absorbing sample, respectively and $\alpha$ is the absorption coefficient. The absorption coefficient $\alpha$ can be expressed as a function of the density of the absorbing species $(N)$ and the absorption cross section $\sigma$. In our case the index $T$ denotes that we describe the triplet density $N_{T}$ and the triplet absorption cross section $\sigma_{T}$.

$$
\alpha_{T}=N_{T} \sigma_{T} .
$$

In the PIA experiment photogenerated triplets act as absorbers, and therefore change the transmission $T$ in this spectral region by an amount $\Delta T$. The transmitted intensity in the region of triplet-triplet (TT) absorption under photoexcitation is denoted as $I_{T}$. $I$ is the transmitted intensity in the same spectral region for the sample in the ground state, i.e., without any triplets present. Now we can use the Eqs. (1) and (2) to express the change in transmission $\Delta T / T$ induced by the photogenerated triplets:

$$
\Delta T / T=\left(I_{T}-I\right) / I=I_{T} / I-1=\exp \left(-N_{T} \sigma_{T} d\right)-1
$$

and we can express the triplet density $N_{T}$ as function of the photoinduced signal.

$$
N_{T}=-\left(\sigma_{T} d\right)^{-1} \ln (\Delta T / T+1) .
$$

We assume $\sigma_{T} \cong 8 \times 10^{-16} \mathrm{~cm}^{2}$, as found for other conjugated molecules, ${ }^{27,28}$ and $d=1 / \alpha=100 \mathrm{~nm}$ to account for the penetration depth of the pump beam with $\alpha$ being $10^{5} \mathrm{~cm}^{-1}$. Since the pump beam does not deposit a significant amount of energy in regions of the samples much deeper than $1 / \alpha$ we assume the depth $d$ of the region within which triplets are generated to be $1 / \alpha$.

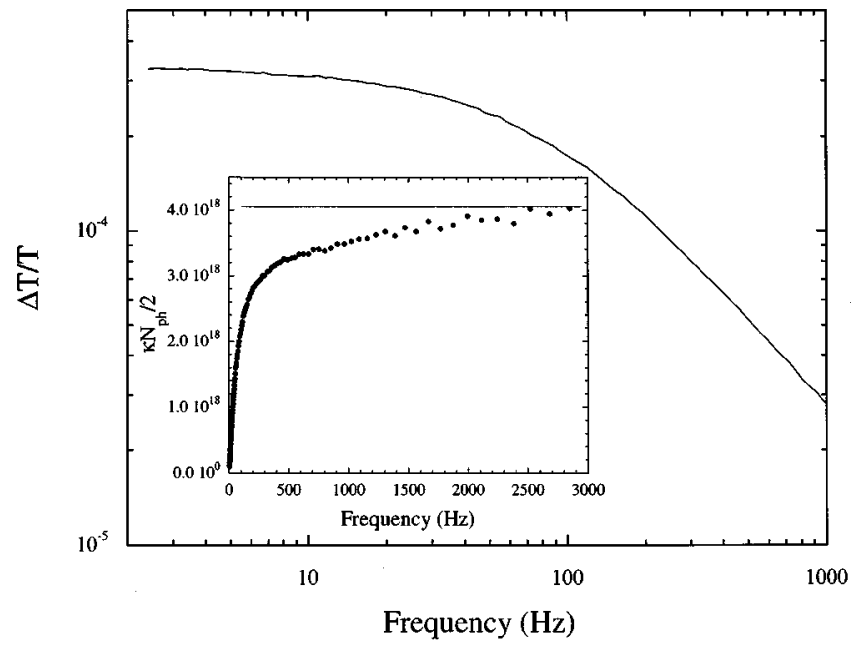

FIG. 7. Photoinduced absorption signal at $1.75 \mathrm{eV}$ as function of the modulation frequency; excitation power $=140 \mathrm{~mW}$. Insert: Calculated value for $\kappa N_{p h} / 2$ versus the modulation frequency.

Figure 6 shows the behavior of the photoinduced absorption peak at $1.75 \mathrm{eV}$ versus the pump intensity. Using Eq. (4), we can convert the obtained $\Delta T / T$ values to the triplet density. As we will discuss and show later (Fig. 7), this value can be taken as the quasi steady state triplet density in spite of the fact that it was obtained in a photomodulation experiment. In order to quantify the triplet density versus the absorbed photons from the pumping power $P$, we can easily calculate the average density of absorbed photons per unit time $\left\langle N_{p h}\right\rangle\left(\mathrm{cm}^{-3} \mathrm{~s}^{-1}\right)$ each one carrying an energy $h c / \lambda$,

$$
\left\langle N_{p h}\right\rangle=P \lambda / h c(0.5 / V)
$$

where $V$ is the film volume corresponding to the laser spot. In the foregoing formula we assume that the reflectivity is roughly $7 \%$ as found for similar materials, ${ }^{29}$ and we measure the transmission through the relevant layer at the pump beam wavelength to be roughly $40 \%$. Both effects reduce the number of absorbed photons to about $50 \%$ of the incident ones. In the insert of Fig. 6, we show the calculated density of triplets versus the absorbed photons. The sublinear behavior shown for the PIA signal in Fig. 6 suggests a significant contribution of a bimolecular decay process. In this way if we consider that the triplets are created by intersystem crossing from the singlet and can decay either by the weakly forbidden recombination to the ground state or by triplettriplet annihilation, we can describe this processes by a simple rate equation, ${ }^{30}$

$$
d N_{T} / d t=\kappa N_{p h}-\beta N_{T}-\gamma N_{T}^{2},
$$

where $\kappa$ is the triplet generation probability, $\beta$ is the triplet decay constant, and $\gamma$ the triplet-triplet annihilation rate constant. For this rate equation we assume that all absorbed photons create singlets which in turn create triplets with a probability $\kappa$. Therefore, the triplet generation term can be described as $\kappa N_{p h}$, with $N_{p h}$ being the number of absorbed photons (singlet excitons) per time unit. Solving Eq. (6) for the steady state we obtain for the triplet density 


$$
N_{T}=-\beta / 2 \gamma+\left[(\beta / 2 \gamma)^{2}+\kappa N_{p h} / \gamma\right]^{1 / 2} .
$$

This equation is a very good approximation for the integral solution over the sample depth $x$ (between 0 and $d$ ) for the mean value of the triplet population,

$$
\left\langle N_{T}\right\rangle=1 / d \int\left\{-\beta / 2 \gamma+\left[(\beta / 2 \gamma)^{2}+\kappa N_{p h}(x) / \gamma\right]^{1 / 2}\right\} d x,
$$

where $N_{p h}(x)$ is the depth-dependent profile of the number of absorbed photons (singlet excitons) per time unit. Equation (7) can be used to fit the curve in the insert of Fig. 6, in this way it is possible to obtain the ratios $\beta / 2 \gamma$ and $\kappa / \gamma$. The values that we get with the fit are $\beta / 2 \gamma=4.2 \times 10^{+16} \mathrm{~cm}^{-3}$ and $\kappa / \gamma=2.1 \times 10^{+12} \mathrm{~cm}^{-3} \mathrm{~s}$. These data are however not sufficient to calculate the rate constants. Therefore we determined the modulation frequency dependence of the PIA signal at fixed excitation density (Fig. 7), which can be used to determine these values. It has been shown recently ${ }^{31}$ that far from the steady-state conditions, i.e., for high-modulation frequencies the triplet density is independent of the decay rate constant and can be expressed as

$$
N_{T}=\kappa N_{p h} / 2 \mathrm{v},
$$

where $v$ is the modulation frequency. The insert of Fig. 7 was obtained by calculating $N_{T}$ from $\Delta T / T$ via Eq. (4). At high-modulation frequencies $v N_{T}$ is constant and equal to $\kappa N_{p h} / 2$ [see Eq. (9)]. Using $N_{p h}=4 \times 10^{21} \mathrm{~cm}^{-3} \mathrm{~s}^{-1}$ at the experimental conditions (140 $\mathrm{mW}$ pump beam power) and the asymptotic value of $\mathrm{v} N_{T}=\kappa N_{p h} / 2=4 \times 10^{18} \mathrm{~cm}^{-3} \mathrm{~s}^{-1}$ at high-modulation frequencies we obtain $\kappa$, and consequently also $\beta$ and $\gamma: \kappa=2 \times 10^{-3}, \quad \gamma=10^{-15} \mathrm{~cm}^{-3} \mathrm{~s}^{-1}$, and $\beta$ $=84 \mathrm{~s}^{-1}$. The value obtained for $\gamma$ is in good agreement with the value found for MEH-PPV. ${ }^{32}$

In order to compare the observed densities of species, we calculate the steady-state singlet exciton density. It is obtained by multiplying the number of absorbed photons $/\left(\mathrm{s} \mathrm{cm}^{3}\right)$ by the lifetime of the singlet excitons $\tau_{P L}$. Using the highest laser intensity in the insert of Fig. 6 we obtain: $200 \mathrm{~mW}$ laser power, $6.0 \times 10^{21}$ photons $/\left(\mathrm{s} \mathrm{cm}^{3}\right)$ at a $\Delta T / T$ of $6.2 \times 10^{-4}$ with a calculated triplet density 7.7 $\times 10^{16}$ triplets $/ \mathrm{cm}^{3}$. To estimate the upper limit of the steadystate singlet exciton density we use the observed $\tau_{P L}$ for the PDHPT solution (see above) and obtain 5 $\times 10^{12}$ singlets $/ \mathrm{cm}^{3}$. However, this upper limit is not reached in solid-state PDHPT samples, since in these films $\tau_{P L}$ is reduced to $160 \mathrm{ps}$ due to rapid nonradiative decay-reducing the expected steady state singlet exciton density to be $(160 / 860) 5 \times 10^{12}=9 \times 10^{11}$ singlets $/ \mathrm{cm}^{3}$. In this way we calculate the ratio of triplet/singlet state densities: (7.7 $\times 10^{16}$ triplets $\left./ \mathrm{cm}^{3}\right) /\left(9 \times 10^{11}\right.$ singlets $\left./ \mathrm{cm}^{3}\right)=9 \times 10^{4}$. These values show clearly that the triplet state is the dominating excited state in PDHPT samples, and can explain the intense TT absorption signal detected with the PIA measurement.

The modulation frequency $(f)$ dependence of the PIA signal at fixed excitation density in Fig. 7 can also be used to determine the lifetime of the triplet states giving rise to it. The influence of a time-constant or lifetime $\tau$ on the signal vector $\mathbf{R}$ measured in a typical optical modulation measurement with a lock in amplifier can be described in the following way:

$$
\mathbf{R}=\frac{\mathbf{c}\left\{\exp \left[i \tan ^{-1}(2 \pi f \tau)\right]\right\}}{\sqrt{1+(2 \pi f \tau)^{2}}}
$$

if a single time constant or lifetime is involved. If two timeconstants are involved, their influence on the signal vector is described in the following equation:

$$
\mathbf{R}=\frac{\mathbf{c}_{1}\left\{\exp \left[i \tan ^{-1}\left(2 \pi f \tau_{1}\right)\right]\right\}}{\sqrt{1+\left(2 \pi f \tau_{1}\right)^{2}}}+\frac{\mathbf{c}_{2}\left\{\exp \left[i \tan ^{-1}\left(2 \pi f \tau_{2}\right)\right]\right\}}{\sqrt{1+\left(2 \pi f \tau_{2}\right)^{2}}},
$$

where $\mathbf{R}$ is the intensity of the signal, $\tau, \tau_{1}$, and $\tau_{2}$ are the lifetimes of the electronic state, and $\mathbf{c}, \mathbf{c}_{1}$ and $\mathbf{c}_{2}$ are the weight of each component. $\mathbf{R}$ has to be written in vector form since it describes both the intensity and the phase of the signal relative to the excitation, i.e., in our case the modulated laser pump beam. If there are two time constants involved, it means that there is an initial branching in the population into two separate uncoupled states, which decay with different decay rates. If the decay takes place from the same state the decay rates will just add up to $k_{\text {total }}=k_{1}+k_{2}$ with the "lifetime" to be $1 / \tau_{\text {total }}=1 / \tau_{1}+1 / \tau_{2}$. The physical meaning is that there is no single state with one lifetime but there are uncoupled states with different lifetimes.

The optical modulation experiment probes the strength of an electronic transition of states with certain lifetimes. We have used the previous equations to model the behavior of the photoinduced absorption depending on the frequency of the chopper wheel. The data could only be modelled properly by using two time-constants, 1.4 and $5.5 \mathrm{~ms}$ with respective weights of 1 and 0.7 , with a weighted average $\tau_{a v}$ $=3 \mathrm{~ms}$. These time constants have to be compared to the decay times given by the parameters $N_{T}, \beta$, and $\gamma$. We obtained $\gamma=10^{-15} \mathrm{~cm}^{3} \mathrm{~s}^{-1}, \quad \beta=84 \mathrm{~s}^{-1}, \quad$ and $N_{T}=6.6$ $\times 10^{-16} \mathrm{~cm}^{-3}$ for $140-\mathrm{mW}$ laser power. The respective decay constants at the particular value of $N_{T}$ are $1 / \beta=12 \mathrm{~ms}$ for the single exponential decay and $1 /\left(N_{T} \gamma\right)=15 \mathrm{~ms}$ for the bimolecular decay. They will add up to show a decay time of $(1 / 12 \mathrm{~ms}+1 / 15 \mathrm{~ms})^{-1}=6.7 \mathrm{~ms}^{32,33}$ Considering that $\gamma$ and $\beta$ are determined by fitting the dependence of the photoinduced absorption on excitation power the agreement with $\tau_{a \mathrm{v}}$ $=3 \mathrm{~ms}$ determined from the data shown in Fig. 7 is highly supportive of the proposed model.

\section{CONCLUSIONS}

We have shown that the high energy optical gap of PDHPT is mainly determined by the large inter-ring torsion induced by the steric hindrance between alkyl groups. By optical excitation, singlet excitons with high interchain mobility are created in PDHPT films, which leads to a biexponential decay behavior. The interchain migration of excitons towards non-radiative centers reduces the emission OY from the $45 \%$ of the solution to the $20 \%$ of the thin film. Furthermore, the introduction of carbonyl groups by UV irradiation increases the number of quenching sites in the film reducing the decay time and the emission QY. 
By means of the PIA measurement we have been able to determine the generation probability $\kappa$; the decay constant $\beta$, and the annihilation constant $\gamma$ of the triplet excitons, and we have shown evidences for the TT annihilation as a significant de-excitation pathway in this material. Based on these data we calculate the ratio of triplet/singlet state densities to be on the order of $10^{5}$.

\section{ACKNOWLEDGMENTS}

We wish to thank F. P. Wenzl for assistance with the doping experiment. The work in Graz was partly supported by the FWF Project No. P 12806-PHY. M. A. Loi and W. Graupner acknowledge the partial financial support from the ASPIRES 1999 program of Virginia Tech.
${ }^{1}$ M. Fahlman, J. Birgersson, K. Kaeriyama, and W. R. Salaneck, Synth. Met. 75, 223 (1995).

${ }^{2}$ Y. Yang, Q. Pei, and J. Heeger, J. Appl. Phys. 79, 934 (1996).

${ }^{3}$ M. Remmers, D. Neher, J. Grüner, R. H. Friend, G. H. Gelinck, J. M. Warman, C. Quattrocchi, D. A. dos Santos, and J.-L. Brédas, Macromolecules 29, 7432 (1996).

${ }^{4}$ N. Tanigaki, H. Masuda, and K. Kaerijama, Polymer 39, 1221 (1997).

${ }^{5}$ Y. S. Liu, P. de Marco, and W. R. Ware, J. Phys. Chem. 97, 5995 (1993).

${ }^{6}$ J. Stampfl, S. Tasch, G. Leising, and U. Scherf, Synth. Met. 71, 2125 (1995).

${ }^{7}$ R. A. J. Janssen, L. Smilowitz, N. S. Sariciftci, and D. Moses, J. Chem. Phys. 101, 1787 (1994).

${ }^{8}$ M. Remmers, B. Müller, K. Martin, H. J. Räder, and W. Köhler, Macromolecules 32, 1073 (1999).

${ }^{9}$ F. Meyers, A. J. Heeger, and J. L. Brédas, J. Chem. Phys. 97, 2750 (1992).

${ }^{10}$ G. Leising, K. Pichler, and F. Stelzer, Electronics Properties of Conjugated Polymers III, edited by H. Kuzmany, S. Roth, M. Mehring, Springer Series in Solid-State Sciences Vol. 91 (Springer, Berlin, 1989), p. 100.

${ }^{11}$ N. Hosaka, H. Tachibana, N. Shiga, M. Matsumoto, and Y. Tokura, Phys. Rev. Lett. 82, 1672 (1999).

${ }^{12}$ Y. Kanemitsu, K. Suzuki, Y. Masumoto, Y. Tomiuchi, Y. Shiraishi, and M. Kuroda, Phys. Rev. B 50, 2301 (1994).

${ }^{13}$ A. Piaggi, R. Tubino, and L. Colombo Phys. Rev. B 51, 1624 (1995).

${ }^{14}$ G. Bongiovanni, M. A. Loi, A. Mura, A. Piaggi, S. Luzzati, and M. Catellani, Chem. Phys. Lett. 288, 749 (1998).

${ }^{15}$ I. D. W. Samuel, G. Rumbles, and C. J. Collison, Phys. Rev. B 52, R11 573 (1995).

${ }^{16}$ J. Cornil, A. J. Heeger, and J. L. Bredas, Chem. Phys. Lett. 272, 463 (1997).

${ }^{17}$ M. Yan, L. J. Rothberg, E. W. Kwock, and T. M. Miller, Phys. Rev. Lett. 75, 1992 (1995).

${ }^{18}$ I. D. W. Samuel, B. Crystall, G. Rumbles, P. L. Burn, A. B. Holmes, and R. H. Friend, Chem. Phys. Lett. 213, 472 (1993).

${ }^{19}$ M. Yan, L. J. Rothberg, F. Papadimitrakopoulos, M. E. Galvin, and T. M. Miller, Phys. Rev. Lett. 73, 744 (1994).
${ }^{20}$ J. C. Scott, J. H. Kaufman, P. J. Brock, R. DiPietro, J. Salem, and J. A. Goitia, J. Appl. Phys. 79, 2745 (1996).

${ }^{21}$ A. Rajagopal, N. Koch, J. Ghijsen, R. L. Johnson, K. Kaeriyama, G. Leising, and J.-J. Pireaux, in print J. Appl. Phys.

${ }^{22}$ D. G. J. Sutherland, J. A. Carlisle, P. Elliker, G. Fox, T. W. Hagler, I. Jimenez, H. W. Lee, K. Pakbaz, L. J. Terminello, S. C. Williams, F. J. Himpsel, D. K. Shuh, W. M. Tong, J. J. Jia, T. A. Callcott, and D. L. Ederer, Appl. Phys. Lett. 68, 2046 (1996).

${ }^{23}$ J. B. Lambert, H. F. Shurvell, D. Lightner, R. G. Cooks, Introduction to Organic Spectroscopy (Macmillan, New York, 1987).

${ }^{24}$ G. Louarn, J. P. Buisson, S. Lefrant, and D. Fichou, J. Phys. Chem. 99, 11399 (1995).

${ }^{25}$ J. A. Stephens, R. H. Friend, M. Remmers, and D. Neher, Synth. Met. 84, 645 (1997).

${ }^{26}$ D. Beljonne, J. Cornil, R. H. Friend, R. A. J. Janssen, and J. L. Brédas, J. Am. Chem. Soc. 118, 6453 (1996).

${ }^{27}$ Z. Vardeny, J. Orenstein and G. L. Baker, J. Phys. (Paris), Colloq. C3 44, 325 (1983).

${ }^{28}$ B. Kraabel, M. Joffre, C. Lapersonne-Meyer, and M. Scott, Phys. Rev. B 58, 15777 (1998).

${ }^{29}$ L. A. A. Pettersson, L. S. Roman, and O. Inganäs, J. Appl. Phys. 86, 487 (1999).

${ }^{30}$ V. Dyakonov and E. Frankevich, Chem. Phys. 227, 203 (1998).

${ }^{31}$ M. Wohlgenannt, W. Graupner, G. Leising, and Z. V. Vardeny, Phys. Rev. Lett. 82, 3344 (1999).

${ }^{32}$ G. D. Hale, S. J. Oldenburg, and N. J. Halas, Phys. Rev. B 55, R16 069 (1997).

${ }^{33}$ R. Sander, V. Herrmann, and R. Menzel, J. Chem. Phys. 104, 4390 (1996).

${ }^{34}$ G. Leising, O. Ekström, W. Graupner, F. Meghadadi, M. Moser, G. Kranzelbinder, T. Jost, S. Tasch, B. Winkler, L. Athouel, G. Froyer, U. Scherf, K. Müllen, G. Lanzani, M. Nisoli, and S. De Silvestri, Proc. SPIE 2852, 189 (1996).

${ }^{35}$ D. Grebner, M. Helbig, and S. Rentsch, J. Phys. Chem. 99, 16991 (1995).

${ }^{36}$ Y. Kanemitsu, N. Shimizu, K. Suzuki, Y. Shiraishi, and M. Kuroda, Phys. Rev. B 54, 2198 (1996).

${ }^{37}$ W. Graupner, F. Meghdadi, G. Leising, G. Lanzani, M. Nisoli, S. De Silvestri, W. Fischer, and F. Stelzer, Phys. Rev. B 56, 10128 (1997). 